\title{
Influence from Polarized Galactic Background Noise on L-band Measurements of the
} Sea Surface Salinity

\section{Søbjærg, Sten Schmidl; Skou, Niels}

\section{Published in:}

Proceedings of IGARSS'04

Link to article, DOI:

10.1109/IGARSS.2004.1368669

Publication date:

2004

Document Version

Publisher's PDF, also known as Version of record

Link back to DTU Orbit

Citation (APA):

Søbjærg, S. S., \& Skou, N. (2004). Influence from Polarized Galactic Background Noise on L-band Measurements of the Sea Surface Salinity. In Proceedings of IGARSS'04 (Vol. 2). IEEE.

https://doi.org/10.1109/IGARSS.2004.1368669

\section{General rights}

Copyright and moral rights for the publications made accessible in the public portal are retained by the authors and/or other copyright owners and it is a condition of accessing publications that users recognise and abide by the legal requirements associated with these rights.

- Users may download and print one copy of any publication from the public portal for the purpose of private study or research.

- You may not further distribute the material or use it for any profit-making activity or commercial gain

- You may freely distribute the URL identifying the publication in the public portal 


\title{
Influence from Polarized Galactic Background Noise on L-band Measurements of the Sea Surface Salinity
}

\author{
Sten Schmidl Søbjærg \& Niels Skou \\ Ørsted-DTU, Electromagnetic Systems, Building 348 \\ Technical University of Denmark \\ DK 2800 Kongens Lyngby, Denmark \\ E-mail: sss@oersted.dtu.dk
}

\begin{abstract}
The polarimetric EMIRAD radiometer, based on novel digital down conversion and detection techniques, has been installed on a C-130 aircraft from the Royal Danish Air Force during the L-band Ocean Salinity Airborne Campaign (LOSAC) in 2001 and 2003. Full $360^{\circ}$ circle flight patterns around the same target area as well as clover leaf patterns have been measured, and both provide an azimuth signature of the ocean at a constant incidence angle.
\end{abstract}

The resulting azimuth signatures show significant variations in all the three first Stokes parameters, and a correlation is found between the downwelling galactic background signal and the measured results. The measured $3^{\text {rd }}$ Stokes parameter has variations of the same order of magnitude as the two linear polarizations, and to verify this result, an experiment for direct observation of the sky over long time is set up. This experiment confirms the presence of a polarized galactic background signal, and conclusions are made with respect to the necessity for polarimetric corrections in future measurements over the sea at L-band.

Keywords-component; L-band; salinity; galactic; polarimetric radiometry

\section{INTRODUCTION}

Two L-band (1.4 GHz) radiometer systems for global ocean salinity mapping are planed for launch in 2007/2008: The SMOS (soil moisture ocean salinity) mission, selected recently for phase C/D studies by ESA, and Aquarius, being prepared by NASA. Measurements of the L-band brightness temperature will provide a sensitivity to salinity, which at best is (ocean with high salinity) DTB / DS $=1 \mathrm{~K} / \mathrm{psu}$ (vertical polarization, $50^{\circ}$ incidence angle). To find salinity to the $0.1 \mathrm{psu}$ level hence requires radiometric measurements to better than $0.1 \mathrm{~K}$, and knowledge concerning the influence from other effects to the same level. Most of these effects have been modeled and understood, and some correction methods have been proposed.

One of the effects, which requires further investigation, is the influence from galactic background noise, being reflected in the sea surface. A map of the galactic background is available, if it is assumed that the signal is fully unpolarized, i.e. the 2nd, the 3rd, and the 4th Stokes parameters are equal to zero, but very few verifications of this assumption exist. Likewise the reflection mechanism at the sea surface is subject for further investigation, and results must be available for different sea states to provide a model.

\section{INFLUENCE FROM THE GALACTIC BACKGROUND}

For a known galactic background and a perfect plain surface, the influence from the downwelling signal is easily calculated from the scalar Fresnel reflection coefficient of the surface. As the reflection mechanism is assumed perfect, only one observation direction contributes, and the total signal is given by (1)

$$
T_{A}=T_{S}+T_{D} \eta
$$

where $T_{A}$ is the apparent temperature at the antenna, $T_{S}$ the brightness temperature of the surface, $T_{D}$ the downwelling radiation from the direction of the reflected beam, and $\eta$ the scalar reflection coefficient. For an antenna with the beam width, $\phi$, the downwelling background radiation must be integrated over the area, covered by the reflected antenna beam. This may be done once, prior to the data correction step, as the covered area will be determined only by the beam shape and the pointing of the beam center. Hence the integrated contribution for each pointing direction may be calculated and stored in a matrix for look-up.

The scalar reflection coefficient is directly related to the surface brightness temperature, as the reflectivity is directly found from one minus the emissivity. For a non-perfect surface, the resulting contribution is blurrier, as the total radiation, reflected towards the antenna, depends on the distribution of pointing directions from the sea surface. Some simple models cam be assumed, however:

- Scalar reflection, given by 1 minus the emissivity

- Scalar reflection with a reduced reflection coefficient

- Distributed reflection within a cone around the pointing direction of the antenna.

It must be assumed that the latter method will provide the best results, but due to the very limited amount of data available, no proper model has been created, and the first two methods will be used. The methods are identical, apart from a modulation factor in front of the expected reflection coefficient. Setting this factor to one, the two methods will be identical. In this paper, 
the reduction factor will be calculated individually for each example, but in practical applications, such as the SMOS mission, a model function, providing the factor as a function of the Sea State, must be available.

\section{RESULTS FROM AIRBORNE MEASUREMENTS}

A series of measurements of the sea surface was carried out within the L-band Ocean Salinity Airborne Campaign, LOSAC, which took place from the spring of 2001 until the fall of 2003. The primary objective of the campaign was to identify eventual variations of the surface brightness temperature as a function of the azimuth observation direction, which is a well known effect at higher frequencies, where it is used for estimation of the sea surface wind direction.

The EMIRAD L-band radiometer [1] was used for the measurements, measuring all four Stokes parameters simultaneously. The radiometer is a direct sampling radiometer, covering the protected band from $1400 \mathrm{MHz}$ to $1427 \mathrm{MHz}$, and providing sensitivity below $100 \mathrm{mK}$ for $1 \mathrm{sec}$. integration. The drift is kept below $50 \mathrm{mK}$ within 15 minutes, thus making it very suitable for the LOSAC measurements.

Corrections have been applied to calibrate the data and to remove the effect of instrument imperfections as well as the influence from variations of the aircraft attitude during data takes $[2,3]$. The resulting signal thus provides azimuth signatures of the sea surface, only including eventual wind effects and the effect from the galactic noise.

The campaign was set up with focus on rapid sampling of the brightness temperature of the same sea surface point from different azimuth directions, and an aircraft installation with a side looking $90 \mathrm{~cm}$ square horn with a constant depression was selected. In this configuration, a circle flight track will provide the desired information, and as the data acquisition time for this track is only a few minutes, constant sea surface conditions during the data take may be assumed. A change of the aircraft bank angle directly changes the incidence angle, and hence a range of incidence angles may be covered within a short flight time. The primary drawback for this flight pattern is the limited observation time for each azimuth direction and incidence angle, thus providing limited data for integration.

As an alternative to the circle tracks, a number of cloverleaf flight patterns, each having eight legs, were performed. This pattern provides a long integration time for each sampling point, but unfortunately it has a series of drawbacks, making it less attractive. First of all it has a poorer sampling in azimuth, but recalling, that higher frequency measurements contain only $1^{\text {st }}$ and $2^{\text {nd }}$ order harmonics [4], eight samples should be sufficient, and the effect of this drawback is reduced. The cloverleaf pattern also takes longer time to obtain due to the maneuvering of the aircraft between the legs, and thus is has a less efficient duty cycle and a larger risk, that the target area may change during the total data take. The cloverleaf pattern is thus selected as secondary pattern, used for verification of the data from the circle tracks.

The results from the individual circle flights show very noisy results, typically with variations above $1 \mathrm{~K}$. Integration of several circles decreases the noise, however, and for the $4^{\text {th }}$
Stokes parameter, the total signal reduction is very near to the square root of the number of integrated circles, strongly indicating, that no deterministic signal is present. For the other Stokes parameters the integration gain is significantly smaller, and for the vertical polarization the strongest deterministic component is identified.

The identified signal is compared to the downwelling galactic background signal, and the result for vertical polarization for a wind speed of $10 \mathrm{~m} / \mathrm{sec}$. is seen in figure 1 . The red curve is the measured signal, the green curve the downwelling signal, offset arbitrarily, and the blue curve the azimuth signature after removal of the downwelling signal, based on a linear regression. The regression coefficient is used to scale the Fresnel reflection coefficient, according to correction method two, mentioned in section 2. For this particular sea state the scaling factor is found to be 0.71 for vertical polarization and 0.73 for horizontal polarization.

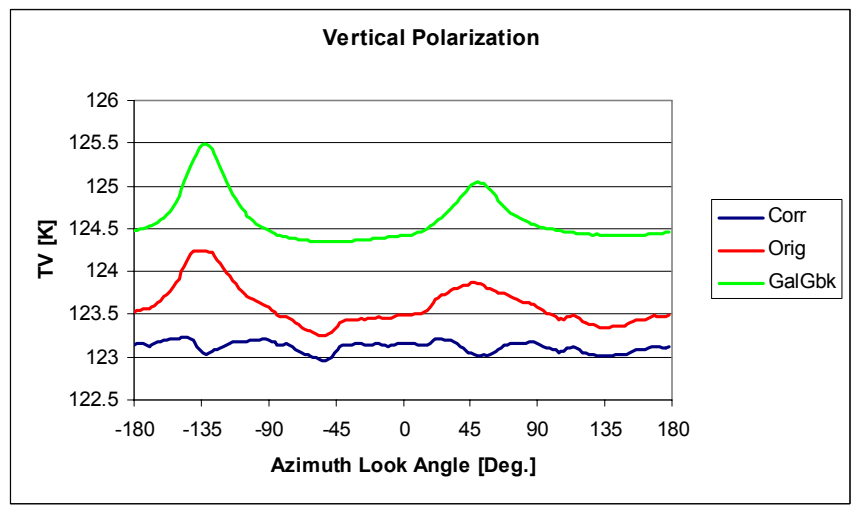

Figure 1. Azimuth signature of the vertical polarized brightness temperature of the ocean surface averaged over 16 individual circle tracks at $10 \mathrm{~m} / \mathrm{sec}$. wind speed. The red curve is the measured data, the green curve the downwelling galactic background radiation offset arbitrarily, and the blue curve is the result after correction.

For the full Stokes vector, the results are shown in figure 2, where only the two linear polarizations are corrected, due to the limited polarimetric data material for the galactic background, and the assumption, that the downwelling signal is unpolarized.
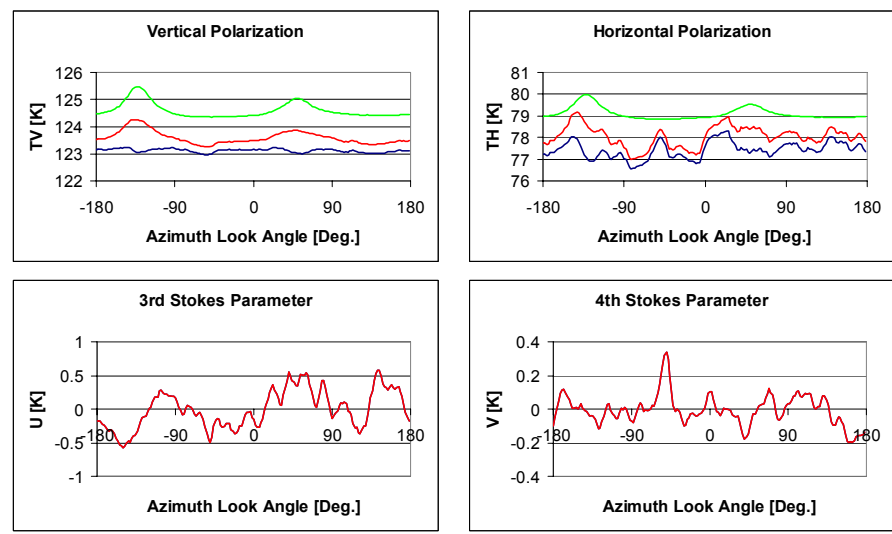

Figure 2. Azimuth signature of the ocean surface averaged over 16 individual circle tracks at $10 \mathrm{~m} / \mathrm{sec}$. wind speed. The red curve is the measured data, the green curve the downwelling galactic background radiation offset arbitrarily, and the blue curve is the result after correction. 
As it is clearly seen from figure 2, the galactic background signal is less clear in the latter three Stokes parameters, and especially for the horizontal polarization and for the $3^{\text {rd }}$ Stokes parameter, it is difficult to make a conclusion due to the presence of noise. To provide better integration, the level flights were added to the data set. These legs provide eight samples of the azimuth signature, enabling for identification of $1^{\text {st }}$ and $2^{\text {nd }}$ order harmonics. These harmonics are plotted in figure 3 , where each parameter is offset to zero mean to fit in the scale.

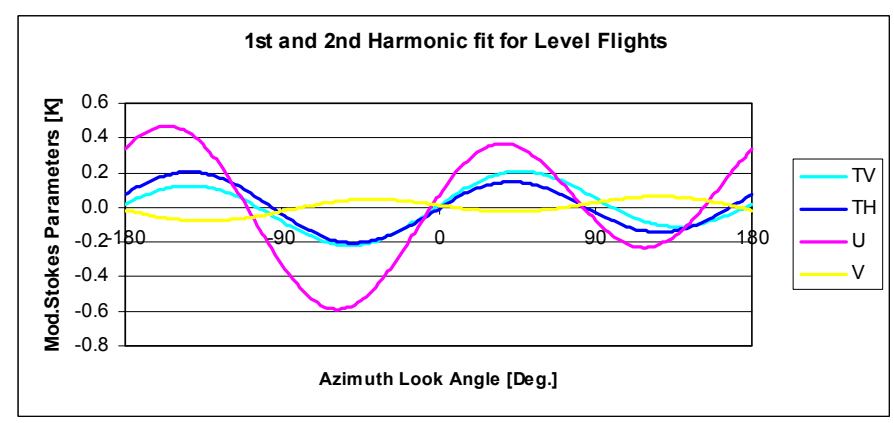

Figure 3. Azimuth signature based on eight level flight tracks, each two minutes long. First and $2^{\text {nd }}$ order harmonics are calculated from the data, and the figure presents these harmonics, offset to zero mean to fit into the scale.

The signatures show a clear harmonic content, and unlike for wind driven sea surface signals [4], the vertical and horizontal polarizations as well as the $3^{\text {rd }}$ Stokes parameter seem to be in phase, with the largest variations in the $3^{\text {rd }}$ Stokes parameter. The maximums occur for approximately the same observation directions as in the circle flights, and this might indicate, that the galactic background also contributes to the $3^{\text {rd }}$ Stokes parameter, i.e. the downwelling signal could seem to be polarized. Only the $4^{\text {th }}$ Stokes parameter is 180 degrees out of phase. As its magnitude is very small, however, around $100 \mathrm{mK}$ peak-to-peak, no final conclusion can be made, if the measured signature originates from the galactic background or is caused by small internal drifts in the radiometer during the 45 minutes data take.

\section{SETUP FOR DIRECT MAESUREMENTS OF THE GALACTIC BACKGROUND}

Based on the experience from the LOSAC campaign, a setup was made for verification of the downwelling galactic background signal. Special emphasis was on measurements of eventual polarimetric signals within the galactic background signal, until now being assumed absent. The EMIRAD polarimetric L-band radiometer was mounted zenith looking at the roof top of the laboratory building, thus observing a constant declination, equal to the latitude of the position. The setup is shown in figure 4.

Due to the practical installation during a long period of time, the instrument could not be left outside, and the whole setup was kept inside the roof top shelter, hence observing the sky through a thin window, which added an offset of a few Kelvin to the signal. The orientation of the antenna's two linear polarizations was given by the shape of the window, and the $\mathrm{V}$ channel was measuring along a vector, pointing to a direction of 15 degrees east. The result of a rotation is a simple polarization mixing of the $2^{\text {nd }}$ and the $3^{\text {rd }}$ Stokes parameters, given by (2), where $\theta$ is the rotation angle.

$\underline{T_{B}^{\prime}}=\left(\begin{array}{c}I^{\prime} \\ Q^{\prime} \\ U^{\prime} \\ V^{\prime}\end{array}\right)=\left(\begin{array}{cccc}1 & 0 & 0 & 0 \\ 0 & \cos (2 \theta) & \sin (2 \theta) & 0 \\ 0 & -\sin (2 \theta) & \cos (2 \theta) & 0 \\ 0 & 0 & 0 & 1\end{array}\right)\left(\begin{array}{l}I \\ Q \\ U \\ V\end{array}\right)=\left(\begin{array}{c}I \\ Q * \cos (2 \theta)+U \sin (2 \theta) \\ -Q^{*} \sin (2 \theta)+U \cos (2 \theta) \\ V\end{array}\right)$

The full Stokes vector will thus contain the full polarimetric information, and alignment to a desired Earth co-ordinate system is easily done by inversion of (2). Two examples of the measured data for a full 24-hour measurement are shown in figure 5 and figure 6 , showing the acquired data on the $20^{\text {th }}$ January 2004, and the $12^{\text {th }}$ of March 2004, respectively. Between the two measurements, the antenna was twisted 90 degrees, inverting the sign of the $3^{\text {rd }}$ Stokes parameters. The horizontal scale represents the time of day, measured from midnight UTC. The green curves correspond to the measured data, and the red curves represent the expected downwelling radiation, found from the unpolarized galactic background map, offset arbitrarily to fit into the scale.

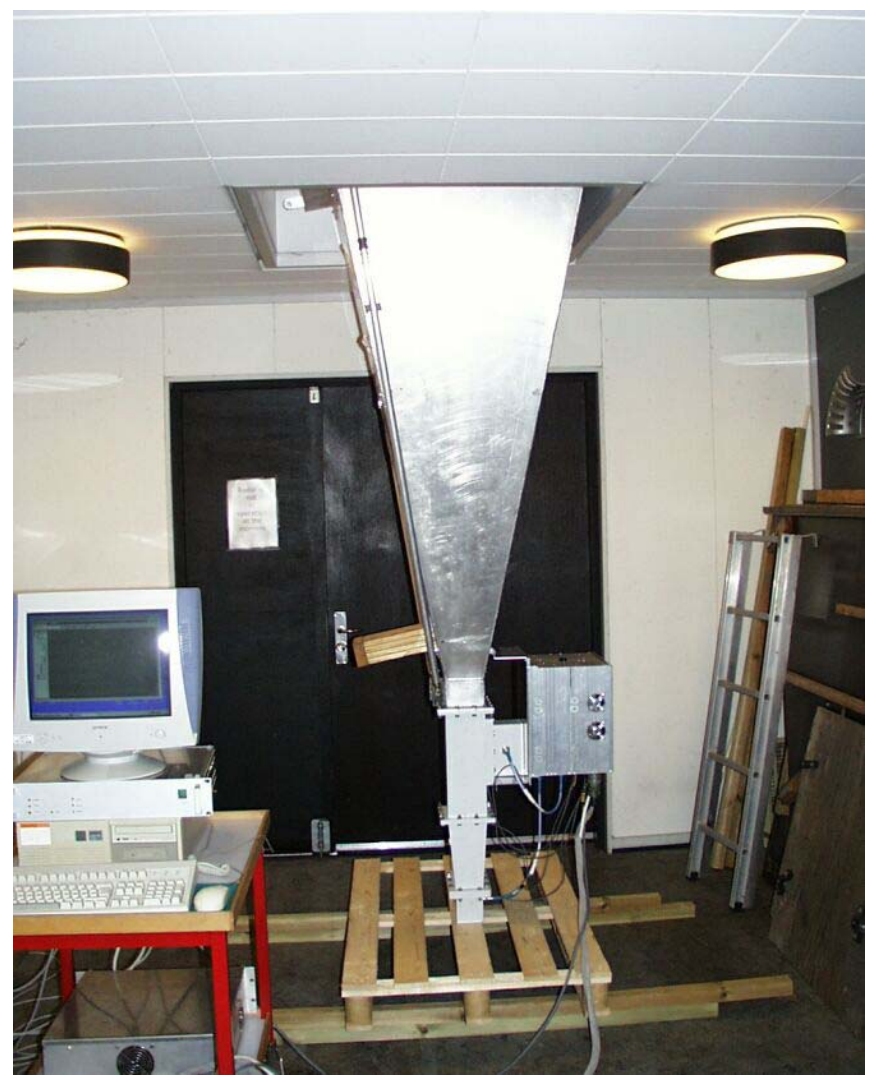

Figure 4. Setup of the EMIRAD L-band radiometer inside the roof-top shelter. The antenna is zenith looking, and it is located behind a small window for long-term measurement.

It is seen clearly from the figures, that the vertical and the horizontal polarizations follow the expected values approximately, although some small deviations are noticed. A short period of sun interference is observed for the $12^{\text {th }}$ of March measurement, but it is easily identified and it can be ignored. The $4^{\text {th }}$ Stokes parameter is around zero all the time, 
and it is seen, that it changes by a maximum of about $50 \mathrm{mK}$. Comparison of its shape to the $4^{\text {th }}$ Stokes parameter for different days shows no correlation, and it may be concluded, that the observed variations are of the order of the accuracy of the radiometer, and probably result from small internal drifts.
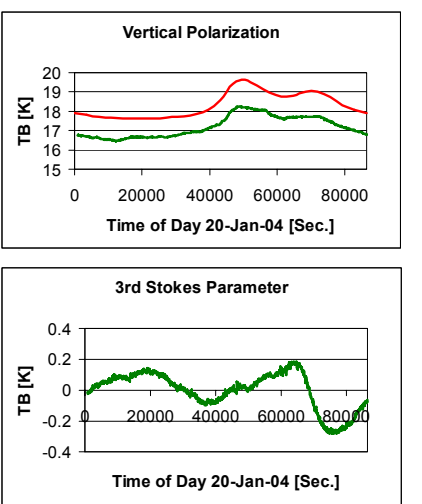
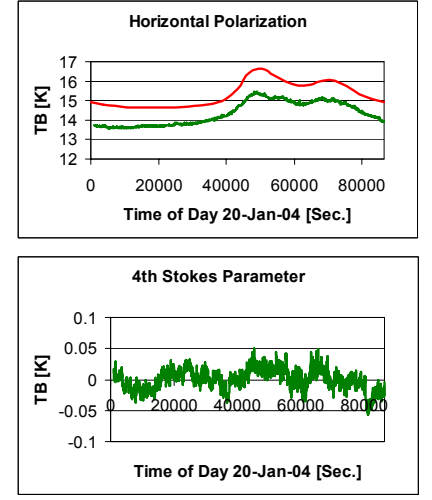

Figure 5. Downwelling signal from the galactic background, measured on January the $20^{\text {th }} 2004$. The antenna is rotated 15 degrees relative to North, and measurements are taken zenith looking at 55 degrees latitude.
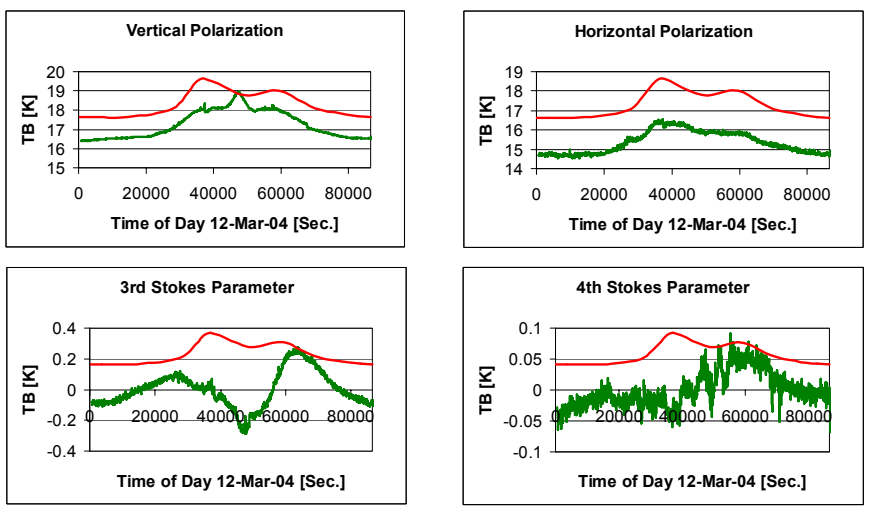

Figure 6. Downwelling signal from the galactic background, measured on March the $12^{\text {th }} 2004$. The antenna is rotated 105 degrees relative to North, and measurements are taken zenith looking at 55 degrees latitude.

The $3^{\text {rd }}$ Stokes parameter, however, has a signal with a maximum variation of $0.5 \mathrm{~K}$, and this signal repeats from day to day with a shift of approximately 4 minutes, equal to the right ascension shift, caused by the movement of the Earth around the Sun. It is thus proven that the effect is not caused by eventual drifts inside the radiometer itself, and the assumption, that the galactic background signal is fully unpolarized, hence does not hold.

Calculation of the true $2^{\text {nd }}$ Stokes parameter from the measured data shows a similar signal, but generally this parameter appears noisier, and moreover it is biased, as the antenna pattern differs between vertical and horizontal polarization. The overall signal variation, however, is also around $0.5 \mathrm{~K}$, and signals of this size must thus also be expected within the circle signatures, measured over the ocean targets.

\section{CONCLUSION}

This paper shows results from airborne polarimetric measurements of the sea surface as well as direct observations of the downwelling galactic background signal. For both types of measurements, it is obvious, that the downwelling signal is partially polarized, and for airborne as well as for space borne sea surface measurements, some signal will be present from the background. Assuming an average reflection coefficient of 0.6, depending on incidence angle and Sea State, a contribution of at least $0.3 \mathrm{~K}$ to the sea brightness temperature, when observing a line at 60 degrees of declination, must be foreseen.

The observed celestial line is almost tangential to the galactic plane, as indicated in figure 5 and figure 6 , where the two crossings are very near. No measurements were made for other declinations, and it is thus not possible to present a full map, useable for correction of the measured flight data. The magnitude of the variations in the $3^{\text {rd }}$ Stokes parameter over a full azimuth scan are similar to the galactic contributions for the linear polarizations, however, and the downwelling signal must be mapped fully, in order to correct the full Stokes vector.

For the space borne missions, an identical signal must be expected, and for any polarimetric measurement, corrections must be applied in order to reach the desired $0.1 \mathrm{~K}$ sensitivity. For SMOS, which is a synthetic aperture radiometer with a synthetic beam of a few degrees, the galactic background influence is even larger, compared to the data in this paper, which are based on the 15 degrees beam width of the EMIRAD radiometer. For the unpolarized galactic background it is well known, that variations up to $20 \mathrm{~K}$ exist at the crossings of the galactic plane, but with the present antenna these signals average to a few Kelvin. It must be expected that a similar effect may be present for the polarimetric signals, and hence errors of several Kelvin may appear. This is fatal for direct applications of the polarimetric signals, but also secondary applications, such as Faraday rotation correction, based on the assumption of zero signal in the $3^{\text {rd }}$ Stokes parameter, will be strongly affected.

\section{ACKNOWLEDGMENT}

Thanks to Dr. David M. LeVine, Goddard Space Flight Center, NASA, for his valuable help with the calculation of the galactic background radiation, as well as for his help providing the unpolarized galactic background map.

\section{REFERENCES}

[1] Jesper Rotbøll, Sten Schmidl Søbjærg and Niels Skou: "A novel L-Band Polarimetric Radiometer featuring Subharmonic Sampling", Radio Science, vol. 38, issue 3, pp MAR11/1-MAR11/7, March 2003.

[2] Sten Schmidl Søbjærg: "Polarimetric Radiometers and their Applications", Ph.D. Thesis, ISBN 87-91184-17-17, Technical University of Denmark, 2002.

[3] "Prime item development specification for the embedded GPS/INS (EGI) H-764", DS34200300 Rev. F, Part I, Honeywell Inc. Military avionics division, 1995.

[4] S. Yueh, "Modeling of wind direction signals in polarimetric sea surface brightness temperatures", IEEE Transactions on Geoscience and Remote Sensing", vol. 35, no. 6, pp. 1400-1418, 1997. 